\title{
Calculation of the amplitudes of elastic waves in anisotropic media in Cartesian or ray-centred coordinates
}

\author{
LUDĚK KLIMES̆
}

Department of Geophysics, Faculty of Mathematics and Physics, Charles University, Ke Karlovu 3, 12116 Praha 2, Czech Republic (http://sw3d.cz/staff/klimes.htm)

Received: July 29, 2018; Revised: October 16, 2018; Accepted: December 12, 2018

\begin{abstract}
We derive various expressions for the amplitude of the ray-theory approximation of elastic waves in heterogeneous anisotropic media, and show their mutual relations. The amplitude of a wavefield with general initial conditions can be expressed in terms of two paraxial vectors of geometrical spreading in Cartesian coordinates, and in terms of the $2 \times 2$ matrix of geometrical spreading in ray-centred coordinates. The amplitude of the Green tensor can be expressed in six different ways: (a) in terms of the paraxial vectors corresponding to two ray parameters in Cartesian coordinates, (b) in terms of the $2 \times 2$ paraxial matrices corresponding to two ray parameters in ray-centred coordinates, (c) in terms of the $3 \times 3$ upper right submatrix of the $6 \times 6$ propagator matrix of geodesic deviation in Cartesian coordinates, (d) in terms of the $2 \times 2$ upper right submatrix of the $4 \times 4$ propagator matrix of geodesic deviation in ray-centred coordinates, (e) in terms of the $3 \times 3$ matrix of the mixed secondorder spatial derivatives of the characteristic function with respect to the source and receiver Cartesian coordinates, and $(f)$ in terms of the $2 \times 2$ matrix of the mixed second-order spatial derivatives of the characteristic function with respect to the source and receiver ray-centred coordinates. The step-by-step derivation of various equivalent expressions, both known or novel, elucidates the mutual relations between these expressions.
\end{abstract}

Keywords: amplitude, transport equation, elastic Green tensor, geodesic deviation, paraxial ray approximation, second--order derivatives of the characteristic function, anisotropy, heterogeneity

\section{INTRODUCTION}

The most important quantity used in ray methods is the travel time defined by means of the non-linear first-order partial differential equation called the HamiltonJacobi equation, which is also often referred to as the eikonal equation in wave propagation problems. The next most important quantity is the zero-order raytheory amplitude defined by means of the transport equation. 


\section{Kliměs}

The solution of the transport equation for the wavefield amplitude is closely related to the equations of geodesic deviation. The Hamiltonian equations of geodesic deviation, also called paraxial ray equations or dynamic ray tracing equations, were proposed by Červený (1972) in order to calculate the wavefield amplitude. The Hamiltonian equations of geodesic deviation have a considerably simpler form than the equivalent Finslerian equations of geodesic deviation (Klimeš, 2013a). The Hamiltonian equations of geodesic deviation were first expressed in general Cartesian coordinates (Červený, 1972), but are also often expressed and solved in the raycentred coordinates connected with a particular ray (Kliměs, 2006b). The solution of the Hamiltonian equations of geodesic deviation describes the coordinate deviations of paraxial rays (paraxial vectors of geometrical spreading) and the slowness-vector deviations of paraxial rays.

The zero-order ray-theory amplitude may be expressed in terms of a solution of particular Hamiltonian equations of geodesic deviation in many ways. Various authors have published various expressions for the zero-order ray-theory amplitude (e.g., Babich, 1961; Červený, 1972; 2001; Kendall and Thomson, 1989; Gajewski

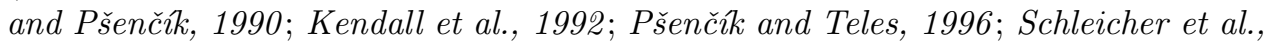
2001; Chapman, 2004; Klimeš, 2006a; 2012; Farra and P̌̌enčik, 2008; Červený and Pšenč $k$, 2014; 2015), and the equivalence of their expressions is not always obvious at first sight. In this paper, we derive various expressions for the zero-order raytheory amplitude. Some of them coincide with the already published expressions, some of them are generalizations of the already published expressions, and some of them are novel.

The zero-order ray-theory amplitude satisfies the transport equation. There are two important kinds of the ray-theory amplitude: the amplitude corresponding to a wavefield with general initial conditions including finite sources, and the amplitude of the Green tensor. Two equivalent but different expressions for the amplitude corresponding to the general initial conditions are presented in Section 4. Twelve equivalent but different expressions for the amplitude of the Green tensor are presented in Section 5. The step-by-step derivation of the equivalent expressions starts with well-known expressions.

For an orthonomic system of rays, corresponding to a wavefield with general initial conditions, we may calculate two paraxial vectors of geometrical spreading corresponding to two ray parameters using the Hamiltonian equations of geodesic deviation. These paraxial vectors may be calculated in Cartesian coordinates or raycentred coordinates. The amplitude of a general wavefield may then be expressed in terms of the paraxial vectors in Cartesian coordinates (Section 4.1), or in terms of the $2 \times 2$ matrix of geometrical spreading in ray-centred coordinates (Section 4.2).

Rays corresponding to the Green tensor are calculated from a point source, and we may again calculate two paraxial vectors of geometrical spreading corresponding to two arbitrary ray parameters at the point source. The amplitude of the Green tensor can then be expressed in terms of the paraxial vectors in Cartesian coordinates (Section 5.3 ), or in terms of the $2 \times 2$ paraxial matrices in ray-centred coordinates (Section 5.2). 
We may also calculate the propagator matrix of geodesic deviation. The propagator matrix may be defined and calculated in Cartesian coordinates or in raycentred coordinates. If we calculate the propagator matrix of geodesic deviation, we may obtain the paraxial vectors using the corresponding initial conditions and the propagator matrix. The paraxial vectors may then be used to determine the amplitude of a general wavefield or the amplitude of the Green tensor as mentioned above.

If we calculate the propagator matrix of geodesic deviation, we may also determine the amplitude of the Green tensor directly from the upper right submatrix of the propagator matrix of geodesic deviation, either from the $3 \times 3$ submatrix in Cartesian coordinates (Section 5.4) or from the $2 \times 2$ submatrix in ray-centred coordinates (Section 5.1).

We may also consider the mixed second-order spatial derivatives of the characteristic function with respect to the source and receiver coordinates. In this case, we may determine the amplitude of the Green tensor using the $3 \times 3$ matrix of these derivatives in Cartesian coordinates (Section 5.6) or the $2 \times 2$ matrix of these derivatives in ray-centred coordinates (Section 5.5).

We do not present the expressions for the amplitude in terms of the surface-tosurface paraxial vectors of geometrical spreading corresponding to two ray parameters, in terms of the $2 \times 2$ surface-to-surface paraxial matrices, or in terms of the upper right $2 \times 2$ submatrix of the $4 \times 4$ surface-to-surface propagator matrix of geodesic deviation, refer to Moser and Červený (200\%). Nor do we demonstrate that the expression for the amplitude of the Green tensor in terms of the three different $2 \times 2$ matrices of the homogeneous second-order spatial derivatives of travel time and the characteristic function with respect to the source and receiver ray-centred coordinates by Červený (2001, Eq. 4.10.43) is applicable to anisotropic media.

The Einstein summation over repetitive lower-case Roman indices, corresponding to the 3 spatial coordinates, is used throughout the paper. Upper-case Roman indices correspond to the first two ray-centred coordinates or to two ray parameters.

\section{RAYS AND GEODESIC DEVIATION}

\subsection{Hamilton-Jacobi equation, travel time, Characteristic function, slowness vector, Hamilton's equations, ray coordinates}

The Hamilton-Jacobi equation is a general partial differential equation of the first order. The Hamilton-Jacobi equation for travel time (action, distance) $\tau\left(x^{m}\right)$ reads

$$
H\left(x^{i}, \frac{\partial \tau}{\partial x^{j}}\left(x^{m}\right)\right)=C
$$

where the function $H\left(x^{i}, p_{j}\right)$ of coordinates $x^{i}$ and of covariant vector $p_{j}$ from the cotangent space at point $x^{i}$ is referred to as the Hamiltonian function. The multivalued solution $\tau\left(x^{m}\right)$ of the Hamilton-Jacobi equation is determined by the initial conditions. 


\section{Kliměs}

The characteristic function (two-point travel time, point-to-point distance) from point $\tilde{x}^{n}$ to point $x^{m}$, denoted by $\tau\left(x^{m}, \tilde{x}^{n}\right)$, is the solution of Hamilton-Jacobi equation (1) with initial conditions $\tau\left(\tilde{x}^{m}, \tilde{x}^{n}\right)=0$.

Gradient

$$
p_{i}=\frac{\partial \tau}{\partial x^{i}}
$$

of the travel time or of the characteristic function is referred to as the slowness vector.

When differentiating the Hamilton-Jacobi equation with respect to coordinates $x^{j}$, we find that the multivalued solution $\tau$ of the Hamilton-Jacobi equation can be calculated along rays (geodesics). Hamilton's equations (equations of rays, ray tracing equations, equations of geodesics) read

$$
\begin{gathered}
\frac{\mathrm{d} x^{i}}{\mathrm{~d} \gamma^{3}}=\frac{\partial H}{\partial p_{i}}\left(x^{m}, p_{n}\right), \\
\frac{\mathrm{d} p_{i}}{\mathrm{~d} \gamma^{3}}=-\frac{\partial H}{\partial x^{i}}\left(x^{m}, p_{n}\right),
\end{gathered}
$$

where $\mathrm{d}$ in the derivatives denotes differentiation along the ray. The meaning of independent parameter $\gamma^{3}$ along the rays depends on the particular form of the Hamiltonian function.

In addition to independent parameter $\gamma^{3}$, corresponding to the Hamiltonian function, we may also parametrize the rays by travel time $\tau$, and define the ray velocity vector

$$
V^{i}=\frac{\mathrm{d} x^{i}}{\mathrm{~d} \tau}
$$

as the derivative of coordinates $x_{i}$ of the ray with respect to travel time $\tau$ along the ray.

In 3-D space, the orthonomic system of rays corresponding to the given initial conditions consists of a two-parametric system of rays which are parametrized by two ray parameters $\gamma^{1}$ and $\gamma^{2}$. The ray parameters together with the independent parameter along the rays form ray coordinates $\gamma^{1}, \gamma^{2}, \gamma^{3}$.

Since the solution of Hamilton-Jacobi equation (1) calculated using Hamilton's equations (3) and (4) is multivalued, it is parametrized by ray coordinates $\gamma^{a}$. Multivalued solution $\tau\left(x^{m}\right)$ or $\tau\left(x^{m}, \tilde{x}^{n}\right)$ is thus expressed as $x^{m}=x^{m}\left(\gamma^{a}\right), \tau=$ $\tau\left(\gamma^{a}\right)$.

\subsection{Ray-centred coordinates}

We may define ray-centred coordinates $q^{a}$ along a particular ray (Klimeš, 2006b). We parametrize the points along the ray by an arbitrary monotonic variable $q^{3}$. At each point $x^{i}\left(q^{3}\right)$ of the ray, we choose two contravariant basis vectors $h_{1}^{i}\left(q^{3}\right)$ and $h_{2}^{i}\left(q^{3}\right)$ perpendicular to slowness vector $p_{i}$,

$$
h_{A}^{i}\left(q^{3}\right) p_{i}=0
$$


Contravariant basis vectors $h_{A}^{i}$ should vary smoothly along the ray. The transformation from the ray-centred coordinates $q^{a}$ to Cartesian coordinates $x^{i}$ is defined by relation

$$
x^{i}=x^{i}\left(q^{3}\right)+h_{A}^{i}\left(q^{3}\right) q^{A} .
$$

Three contravariant basis vectors of the ray-centred coordinate system are

$$
h_{a}^{i}=\frac{\partial x^{i}}{\partial q^{a}}
$$

In matrix notation, we shall denote the first two contravariant basis vectors $h_{1}^{i}$ and $h_{2}^{i}$ as $\mathbf{h}_{1}$ and $\mathbf{h}_{2}$. The slowness vector in ray-centred coordinates at the central ray then reads

$$
p_{a}^{(q)}=p_{i} h_{a}^{i}
$$

Three covariant basis vectors of the ray-centred coordinate system are

$$
\hat{h}_{i}^{a}=\frac{\partial q^{a}}{\partial x^{i}}
$$

In matrix notation, we shall denote the first two covariant basis vectors $\hat{h}_{i}^{1}$ and $\hat{h}_{i}^{2}$ as $\hat{\mathbf{h}}^{1}$ and $\hat{\mathbf{h}}^{2}$.

$$
\begin{aligned}
& 2.3 \text {. Paraxial ray matrices } \\
& \text { of an orthonomic system of rays }
\end{aligned}
$$

For an orthonomic system of rays corresponding to the given initial conditions, we define the $3 \times 3$ paraxial matrix

$$
X_{a}^{i}=\frac{\partial x^{i}}{\partial \gamma^{a}}
$$

of geometrical spreading in Cartesian coordinates. We analogously define the $3 \times 3$ paraxial matrix

$$
Y_{i a}=\frac{\partial p_{i}}{\partial \gamma^{a}}
$$

describing the paraxial slowness vectors in Cartesian coordinates.

The third columns of the paraxial matrices can be obtained from the solution of Hamilton's equations (3) and (4). We shall refer to the first two columns $X_{1}^{i}, X_{2}^{i}$ or $Y_{i 1}, Y_{i 2}$ of the paraxial matrices as the paraxial vectors, and denote them as $\mathbf{X}_{1}$, $\mathbf{X}_{2}$ or $\mathbf{Y}_{1}, \mathbf{Y}_{2}$ in matrix notation. The paraxial vectors can be calculated using the Hamiltonian equations of geodesic deviation (paraxial ray equations, dynamic ray tracing equations), which can be obtained by differentiating Hamilton's equations (3) and (4) with respect to ray coordinates $\gamma^{a}$ (Červený, 1972).

We also define the analogous paraxial matrices

$$
Q_{a}^{i}=\frac{\partial q^{i}}{\partial \gamma^{a}}
$$

and

$$
P_{i a}=\frac{\partial p_{i}^{(q)}}{\partial \gamma^{a}}
$$

in ray-centred coordinates. 


\section{Kliměs}

For fixed ray parameters $\gamma^{A}$, we have $q^{I}=0$ and $p_{I}^{(q)}=0$, see definition (7) and definition (9) with (6). Then

$$
Q_{3}^{I}=0
$$

and

$$
P_{I 3}=0 \text {. }
$$

In ray-centred coordinates, we can thus calculate just the $2 \times 2$ paraxial matrices $Q_{A}^{I}$ and $P_{I A}$ using the Hamiltonian equations of geodesic deviation. In matrix notation, we shall denote these $2 \times 2$ paraxial matrices $\mathbf{Q}$ and $\mathbf{P}$.

We compare definitions (5) and (11), and obtain relation

$$
X_{3}^{k}=V^{k} \frac{\mathrm{d} \tau}{\mathrm{d} \gamma^{3}}
$$

Definition (13) yields identity

$$
Q_{3}^{k}=\delta_{3}^{k} \frac{\mathrm{d} q^{3}}{\mathrm{~d} \gamma^{3}}
$$

2.4. Propagator matrices of geodesic deviation

The propagator matrices of geodesic deviation represent the solution of the Hamiltonian equations of geodesic deviation with identity initial conditions, both in Cartesian coordinates and in ray-centred coordinates (Kendall et al., 1992; Klimeř, 1994).

The $6 \times 6$ propagator matrix of geodesic deviation in Cartesian coordinates may also be defined as the matrix of the derivatives of $x^{i}$ and $p_{i}$ with respect to their initial conditions $\tilde{x}^{j}$ and $\tilde{p}_{j}$ for fixed $\gamma^{3}$. In this paper, we shall use just the $3 \times 3$ upper right submatrix

$$
X_{2}^{i j}=\frac{\partial x^{i}}{\partial \tilde{p}_{j}}
$$

of this propagator matrix. The partial derivatives are calculated for fixed $\gamma^{3}$.

The $6 \times 6$ propagator matrix of geodesic deviation in ray-centred coordinates may also be defined as the matrix of the derivatives of $q^{i}$ and $p_{i}^{(q)}$ with respect to their initial conditions $\tilde{q}^{j}$ and $\tilde{p}_{j}^{(q)}$ for fixed $\gamma^{3}$.

As the consequence of identities (15) and (16), we may also define the $4 \times 4$ propagator matrix of geodesic deviation in ray-centred coordinates as the matrix of the derivatives of $q^{I}$ and $p_{I}^{(q)}$ with respect to their initial conditions $\tilde{q}^{J}$ and $\tilde{p}_{J}^{(q)}$ for fixed $\gamma^{3}$. In this paper, we shall use just the upper right $2 \times 2$ submatrix

$$
Q_{2}^{I J}=\frac{\partial q^{I}}{\partial \tilde{p}_{J}^{(q)}}
$$

of this propagator matrix. In matrix notation, we shall denote this $2 \times 2$ matrix as $\mathbf{Q}_{2}$. 
Calculation of the amplitudes of elastic waves in anisotropic media

\section{AMPLITUDE}

\subsection{Transport equation}

Multivalued zero-order ray-theory amplitude $A=A\left(x_{m}\right)$ of a general elastic wavefield satisfies transport equation

$$
\frac{\partial}{\partial x_{i}}\left(A^{2} \varrho V^{i}\right)=0
$$

(Kliměs, 2006a, Eq. 10), where ray velocity vector $V^{i}$ is given by definition (5).

Function $\varrho=\varrho\left(x_{m}, f_{\kappa}\right)$ is a function parametrizing the transport equation. If $A$ is the amplitude of the displacement of an elastic wavefield, $\varrho$ is the density. From the point of view of differential geometry, amplitude $A$ is a scalar if $\varrho$ is the scalar density of weight -1 (scalar per volume).

The solution of transport equation (21) can be calculated separately along each ray (Babich, 1961; Kliměs, 2006a).

\subsection{Phase shift due to caustics}

Transport equation (21) is a partial differential equation for the square $A^{2}$ of the amplitude, not for the amplitude itself. Even if the solution $A^{2}$ of transport equation (21) is real-valued, amplitude $A$ becomes complex-valued if its square $A^{2}$ becomes negative. Amplitude $A$ is thus complex-valued. Since the complex-valued square root has two branches, it is difficult to determine amplitude $A$ from its square $A^{2}$. We must determine which branch of the amplitude calculated along the ray is correct.

We thus separate square root $A=\sqrt{A^{2}}$ into complex modulus $|A|=\sqrt{\left|A^{2}\right|}$ and complex argument $\exp (\mathrm{i} \varphi)$,

$$
A=|A| \exp (\mathrm{i} \varphi) .
$$

Quantity $\varphi$ in expression (22) is the phase shift due to caustics.

If the rays are real-valued, there is a real-valued solution of transport equation (21) for the square $A^{2}$ of the amplitude. The phase shift due to caustics is then constant in the regions of equal sign of $A^{2}$, and changes by an integer multiple of $\pi / 2$ at the boundaries between the regions.

The calculation of amplitude $A$ along the ray is thus composed of the calculation of its complex modulus $|A|$ along the ray and of the determination of the phase shift $\varphi$ due to caustics. This paper is devoted to the the calculation of the complex modulus $|A|$ of the amplitude.

The rules for the determination of the phase shift due to caustics along the ray were proposed by Lewis (1965), Orlov (1981), Kravtsov and Orlov (1993; 1999), Bakker (1988), Garmany (2001) and Kliměs (2010; 2014).

For an orthonomic system of rays corresponding to a wavefield with general initial conditions, the phase shift due to caustics may be determined using the $3 \times 3$ paraxial matrices in Cartesian coordinates with their derivatives (Kliměs, 2014, Secs 2.2 and 3.2), or using the $2 \times 2$ paraxial matrices in ray-centred coordinates with their derivatives (Kliměs, 2014, Secs 2.1 and 3.1). 


\section{Kliměs}

The phase shift of the Green tensor due to caustics may be determined using the $3 \times 3$ right-hand submatrices of the $6 \times 6$ propagator matrix of geodesic deviation in Cartesian coordinates with their derivatives (Kliměs, 2010, Secs 2.2 and 3.2), or using the $2 \times 2$ right-hand submatrices of the $4 \times 4$ propagator matrix of geodesic deviation in ray-centred coordinates with their derivatives (Kliměs, 2010, Secs 2.1 and 3.1).

\section{AMPLITUDE OF A GENERAL WAVEFIELD}

\subsection{Amplitude in terms of the matrix of geometrical spreading in Cartesian coordinates}

The solution of transport equation (21) may be expressed in various forms. The square of the complex-valued amplitude may be expressed as

$$
A^{2}=C^{2} \frac{1}{\varrho} \frac{\mathrm{d} \tau}{\mathrm{d} \gamma_{3}} \frac{1}{\operatorname{det}\left(X_{a}^{i}\right)}
$$

(Klimeř, 2006a, Eqs 12, 34), where the $3 \times 3$ paraxial matrix $X_{a}^{i}$ of geometrical spreading in Cartesian coordinates is given by definition (11).

This equation represents the generalization of the analogous equation by Babich (1961, Eq. 3.7) from a homogeneous Hamiltonian function to a general Hamiltonian function.

The complex-valued amplitude then reads

$$
A=C \sqrt{\frac{1}{\varrho} \frac{\mathrm{d} \tau}{\mathrm{d} \gamma_{3}} \frac{1}{\left|\operatorname{det}\left(X_{a}^{i}\right)\right|}} \exp (\mathrm{i} \varphi)
$$

where $\varphi$ is the phase shift due to caustics.

Complex-valued factor $C=C\left(\gamma^{1}, \gamma^{2}\right)$ is constant along the ray in a smooth medium and is determined by the initial conditions. It is often referred to as the reduced amplitude (Červený et al., 1988, Eq. 5.19). Reduced amplitude $C$ depends on the selection of ray parameters $\gamma^{1}$ and $\gamma^{2}$.

We insert relation (17) into expression (24) and arrive at expression

$$
A=\frac{C}{\sqrt{\varrho\left|\varepsilon_{i j k} X_{1}^{i} X_{2}^{j} V^{k}\right|}} \exp (\mathrm{i} \varphi)
$$

(Gajewski and Pšenčik, 1990, Eq. 7; Kendall et al., 1992, Eqs 3-4), where paraxial vectors $X_{1}^{i}$ and $X_{2}^{i}$ represent the first two columns of the $3 \times 3$ paraxial matrix (11) of geometrical spreading.

For the special case of a homogeneous Hamiltonian function, paraxial vectors $\mathbf{X}_{1}$ and $\mathbf{X}_{2}$ are tangent to the wavefront, and their cross product is thus normal to the wavefront,

$$
\varepsilon_{i j k} X_{1}^{i} X_{2}^{j}= \pm\left|\mathbf{X}_{1} \times \mathbf{X}_{2}\right| v p_{k}
$$


In this case,

$$
A=\frac{C}{\sqrt{\varrho v\left|\mathbf{X}_{1} \times \mathbf{X}_{2}\right|}} \exp (\mathrm{i} \varphi)
$$

(Červený, 1972, Eq. 29b; Kendall and Thomson, 1989, Eqs 29-30). This equation is not applicable to a general Hamiltonian function.

4.2. Amplitude in terms of the matrix

of geometrical spreading in ray-centred coordinates

Definition (11) yields relation

$$
X_{a}^{k}=\frac{\partial x^{k}}{\partial q^{i}} \frac{\partial q^{i}}{\partial \gamma^{a}}
$$

for the transformation from ray-centred coordinates to Cartesian coordinates. Considering definitions (8) and (13), relation (28) reads

$$
X_{a}^{k}=h_{i}^{k} Q_{a}^{i}
$$

which implies relation

$$
\left|\operatorname{det}\left(X_{a}^{g}\right)\right|=\left|\operatorname{det}\left(h_{i}^{g}\right)\right|\left|\operatorname{det}\left(Q_{a}^{i}\right)\right|
$$

for the determinants. The determinant of the transformation matrix (8) from raycentred to Cartesian coordinates is

$$
\left|\operatorname{det}\left(h_{a}^{i}\right)\right|=\left|\varepsilon_{i j k} h_{1}^{i} h_{2}^{j} h_{3}^{k}\right| .
$$

Since contravariant basis vectors $\mathbf{h}_{1}$ and $\mathbf{h}_{2}$ of the ray-centred coordinate system are tangent to the wavefront, their cross product is normal to the wavefront, and

$$
\varepsilon_{i j k} h_{1}^{i} h_{2}^{j}= \pm\left|\mathbf{h}_{1} \times \mathbf{h}_{2}\right| v p_{k}
$$

We insert relation

$$
h_{3}^{k}=\frac{\partial x^{k}}{\partial q^{3}}
$$

following from definitions (7) and (8), into relation (31) with (32) and obtain relation

$$
\left|\operatorname{det}\left(h_{i}^{g}\right)\right|=\left|\mathbf{h}_{1} \times \mathbf{h}_{2}\right| v \frac{\mathrm{d} \tau}{\mathrm{d} q^{3}}
$$

Relation (30) with relation (34) reads

$$
\left|\operatorname{det}\left(X_{a}^{i}\right)\right|=\left|\mathbf{h}_{1} \times \mathbf{h}_{2}\right|\left|\operatorname{det}\left(Q_{a}^{i}\right)\right| v \frac{\mathrm{d} \tau}{\mathrm{d} q^{3}} .
$$

Due to identities (15) and (18), we have relation

$$
\operatorname{det}\left(Q_{a}^{i}\right)=\operatorname{det}\left(Q_{A}^{I}\right) \frac{\mathrm{d} q^{3}}{\mathrm{~d} \gamma^{3}}
$$

We insert relation (35) with relation (36) into expression (24) and arrive at expression

$$
A(\mathbf{x}, \tilde{x})=\frac{C}{\sqrt{\varrho v\left|\mathbf{h}_{1} \times \mathbf{h}_{2}\right|\left|\operatorname{det}\left(Q_{A}^{I}\right)\right|}} \exp (\mathrm{i} \varphi)
$$

(Kliměs, 2012, Eqs 7, 9) for the amplitude of a general wavefield. 


\section{Kliměs}

\section{AMPLITUDE OF THE GREEN TENSOR}

5.1. Amplitude in terms of the propagator matrix of geodesic deviation in ray-centred coordinates

Using the representation theorem for elastic waves and relation (37) in raycentred coordinates, we can derive expression

$$
A^{\mathrm{G}}(\mathbf{x}, \tilde{\mathbf{x}})=\frac{1}{4 \pi} \frac{1}{\sqrt{\varrho(\mathbf{x}) v(\mathbf{x}) \varrho(\tilde{\mathbf{x}}) v(\tilde{\mathbf{x}})} L(\mathbf{x}, \tilde{\mathbf{x}})} \exp [\mathrm{i} \varphi(\mathbf{x}, \tilde{\mathbf{x}})]
$$

(Červený, 2001, Eq. 5.4.24; Klimě̌, 2012, Eq. 55) for the amplitude of the Green tensor from point $\tilde{\mathbf{x}}$ to point $\mathbf{x}$ in the frequency domain. Here

$$
L(\mathbf{x}, \tilde{\mathbf{x}})=\sqrt{\left|\mathbf{h}_{1}(\mathbf{x}) \times \mathbf{h}_{2}(\mathbf{x})\right|\left|\operatorname{det}\left[\mathbf{Q}_{2}(\mathbf{x}, \tilde{\mathbf{x}})\right]\right|\left|\mathbf{h}_{1}(\tilde{\mathbf{x}}) \times \mathbf{h}_{2}(\tilde{\mathbf{x}})\right|}
$$

(Klimeř, 2012, Eq. 13) is the relative geometrical spreading defined by Červený (2001, Eq. 4.14.45). Amplitude (38) corresponds to the Fourier transform

$$
G_{i k}(\mathbf{x}, \tilde{\mathbf{x}}, \omega)=\int \mathrm{d} t G_{i k}(\mathbf{x}, \tilde{\mathbf{x}}, t) \exp (\mathrm{i} \omega t)
$$

of the Green tensor from time $t$ to circular frequency $\omega$. If the right-hand side of the Fourier transform includes multiplicative factor $(2 \pi)^{-\frac{1}{2}}$ or $(2 \pi)^{-1}$, the right-hand side of expression (38) should be multiplied by the same factor.

The determinant of the transformation matrix (10) from Cartesian to ray-centred coordinates is

$$
\left|\operatorname{det}\left(\hat{h}_{a}^{i}\right)\right|=\left|\varepsilon^{a b c} \hat{h}_{a}^{1} \hat{h}_{b}^{2} \hat{h}_{c}^{3}\right|
$$

Since the covariant basis vectors $\hat{\mathbf{h}}^{1}$ and $\hat{\mathbf{h}}^{2}$ of the ray-centred coordinate system are perpendicular to the ray, their cross product is tangent to the ray, and

$$
\varepsilon^{a b c} \hat{h}_{a}^{1} \hat{h}_{b}^{2}= \pm\left|\hat{\mathbf{h}}^{1} \times \hat{\mathbf{h}}^{2}\right| V^{-1} V^{c}
$$

analogous to relation (32) for the contravariant basis vectors. Here $V$ is the ray velocity and $V^{c}$ is the ray-velocity vector. We insert definition (5) and part

$$
\hat{h}_{i}^{3}=\frac{\partial q^{3}}{\partial x^{i}}
$$

of definition (10) into relation (41) with (42) and obtain relation

$$
\hat{h}_{c}^{3} V^{c}=\frac{\mathrm{d} q^{3}}{\mathrm{~d} \tau} .
$$

Inserting (42) into (41) and considering (44), we arrive at

$$
\left|\operatorname{det}\left(\hat{h}_{a}^{i}\right)\right|=\left|\hat{\mathbf{h}}^{1} \times \hat{\mathbf{h}}^{2}\right| V^{-1} \frac{\mathrm{d} q^{3}}{\mathrm{~d} \tau} .
$$

Since

$$
\left|\operatorname{det}\left(h_{i}^{a}\right)\right|\left|\operatorname{det}\left(\hat{h}_{b}^{j}\right)\right|=1,
$$


relations (34) and (45) yield identity

$$
\left|\mathbf{h}_{1} \times \mathbf{h}_{2}\right|=\left|\hat{\mathbf{h}}^{1} \times \hat{\mathbf{h}}^{2}\right|^{-1} V v^{-1},
$$

which can be inserted into expression (39), both at point $\tilde{\mathbf{x}}$ or point $\mathbf{x}$, e.g.,

$$
L(\mathbf{x}, \tilde{\mathbf{x}})=\sqrt{\left|\mathbf{h}_{1}(\mathbf{x}) \times \mathbf{h}_{2}(\mathbf{x})\right|\left|\operatorname{det}\left[\mathbf{Q}_{2}(\mathbf{x}, \tilde{\mathbf{x}})\right]\right|\left|\hat{\mathbf{h}}^{1}(\tilde{\mathbf{x}}) \times \hat{\mathbf{h}}^{2}(\tilde{\mathbf{x}})\right|^{-1} V(\tilde{\mathbf{x}})[v(\tilde{\mathbf{x}})]^{-1}},
$$

or

$$
L(\mathbf{x}, \tilde{\mathbf{x}})=\sqrt{\frac{\left|\operatorname{det}\left[\mathbf{Q}_{2}(\mathbf{x}, \tilde{\mathbf{x}})\right]\right|}{\left|\hat{\mathbf{h}}^{1}(\mathbf{x}) \times \hat{\mathbf{h}}^{2}(\mathbf{x})\right|\left|\hat{\mathbf{h}}^{1}(\tilde{\mathbf{x}}) \times \hat{\mathbf{h}}^{2}(\tilde{\mathbf{x}})\right|} \frac{V(\mathbf{x})}{v(\mathbf{x})} \frac{V(\tilde{\mathbf{x}})}{v(\tilde{\mathbf{x}})}} .
$$

\subsection{Amplitude in terms of the paraxial matrices in ray-centred coordinates}

Paraxial matrices $\mathbf{Q}(\mathbf{x})$ and $\mathbf{P}(\tilde{\mathbf{x}})$ corresponding to arbitrarily parametrized rays from a point source at $\tilde{\mathbf{x}}$ are related by equation

$$
\mathbf{Q}(\mathbf{x})=\mathbf{Q}_{2}(\mathbf{x}, \tilde{\mathbf{x}}) \mathbf{P}(\tilde{\mathbf{x}})
$$

Inserting relation (50) into expression (39), we obtain expression

$$
L(\mathbf{x}, \tilde{\mathbf{x}})=\sqrt{\left|\mathbf{h}_{1}(\mathbf{x}) \times \mathbf{h}_{2}(\mathbf{x})\right||\operatorname{det}[\mathbf{Q}(\mathbf{x})]||\operatorname{det}[\mathbf{P}(\tilde{\mathbf{x}})]|^{-1}\left|\mathbf{h}_{1}(\tilde{\mathbf{x}}) \times \mathbf{h}_{2}(\tilde{\mathbf{x}})\right|}
$$

for the relative geometrical spreading.

We may insert identity (47) into expression (51), both at point $\tilde{\mathbf{x}}$ or point $\mathbf{x}$, e.g.,

$$
L(\mathbf{x}, \tilde{\mathbf{x}})=\sqrt{\frac{\left|\mathbf{h}_{1}(\mathbf{x}) \times \mathbf{h}_{2}(\mathbf{x})\right||\operatorname{det}[\mathbf{Q}(\mathbf{x})]|}{|\operatorname{det}[\mathbf{P}(\tilde{\mathbf{x}})]|\left|\hat{\mathbf{h}}^{1}(\tilde{\mathbf{x}}) \times \hat{\mathbf{h}}^{2}(\tilde{\mathbf{x}})\right|} \frac{V(\tilde{\mathbf{x}})}{v(\tilde{\mathbf{x}})}},
$$

or

$$
L(\mathbf{x}, \tilde{\mathbf{x}})=\sqrt{\frac{|\operatorname{det}[\mathbf{Q}(\mathbf{x})]|}{\left|\hat{\mathbf{h}}^{1}(\mathbf{x}) \times \hat{\mathbf{h}}^{2}(\mathbf{x})\right||\operatorname{det}[\mathbf{P}(\tilde{\mathbf{x}})]|\left|\hat{\mathbf{h}}^{1}(\tilde{\mathbf{x}}) \times \hat{\mathbf{h}}^{2}(\tilde{\mathbf{x}})\right|} \frac{V(\mathbf{x})}{v(\mathbf{x})} \frac{V(\tilde{\mathbf{x}})}{v(\tilde{\mathbf{x}})}} .
$$

5.3. Amplitude in terms of the paraxial vectors in Cartesian coordinates

We supplement paraxial vectors $Y_{i 2}$ and $Y_{i 1}$ with slowness vector $p_{i}$ to create a $3 \times 3$ matrix. The transformation of this matrix from ray-centred coordinates to Cartesian coordinates reads

$$
\left(\begin{array}{lll}
Y_{i 1} & Y_{i 2} & p_{i}
\end{array}\right)=\hat{h}_{k}^{i}\left(\begin{array}{lll}
P_{k 1} & P_{k 2} & \delta_{k 3} \frac{\mathrm{d} \tau}{\mathrm{d} q^{3}}
\end{array}\right) .
$$

It follows that the corresponding determinants are transformed as

$$
\left|\varepsilon^{i j k} Y_{i 1} Y_{j 2} p_{k}\right|=\left|\operatorname{det}\left(\hat{h}_{k}^{i}\right)\right|\left|\varepsilon^{m n 3} P_{m 1} P_{n 2}\right| \frac{\mathrm{d} \tau}{\mathrm{d} q^{3}} .
$$

We insert relation (45) into relation (55) and obtain

$$
\left|\varepsilon^{i j k} Y_{i 1} Y_{j 2} p_{k}\right|=\left|\hat{\mathbf{h}}^{1} \times \hat{\mathbf{h}}^{2}\right|\left|\operatorname{det}\left(P_{I A}\right)\right| V^{-1} .
$$




\section{Kliměs}

Relations (17), (35) and (36) yield relation

$$
\left|\varepsilon_{i j k} X_{1}^{i} X_{2}^{j} V^{k}\right|=\left|\mathbf{h}_{1} \times \mathbf{h}_{2}\right|\left|\operatorname{det}\left(Q_{A}^{I}\right)\right| v \quad .
$$

We insert relations (56) and (57) into expression (52) and obtain expression

$$
L(\mathbf{x}, \tilde{\mathbf{x}})=\sqrt{\frac{\left|\varepsilon_{i j k} X_{1}^{i}(\mathbf{x}) X_{2}^{j}(\mathbf{x}) V^{k}(\mathbf{x})\right|}{v(\mathbf{x})\left|\varepsilon^{l m n} Y_{l 1}(\tilde{\mathbf{x}}) Y_{m 2}(\tilde{\mathbf{x}}) p_{n}(\tilde{\mathbf{x}})\right| v(\tilde{\mathbf{x}})}}
$$

(Červený and P̌senčik, 2015, Eq. 52) for the relative geometrical spreading. Since vectors $Y_{i 1}$ and $Y_{i 2}$ are tangent to the slowness surface at point $\tilde{\mathbf{x}}$, their cross product is normal to the slowness surface, and

$$
\varepsilon^{i j k} Y_{i 1}(\tilde{\mathbf{x}}) Y_{j 2}(\tilde{\mathbf{x}})= \pm\left|\mathbf{Y}_{1}(\tilde{\mathbf{x}}) \times \mathbf{Y}_{2}(\tilde{\mathbf{x}})\right| V^{k}(\tilde{\mathbf{x}}) V^{-1}(\tilde{\mathbf{x}})
$$

We multiply relation (59) by the slowness vector and obtain

$$
\left|\varepsilon^{i j k} Y_{i 1}(\tilde{\mathbf{x}}) Y_{j 2}(\tilde{\mathbf{x}}) p_{k}(\tilde{\mathbf{x}})\right|=\left|\mathbf{Y}_{1}(\tilde{\mathbf{x}}) \times \mathbf{Y}_{2}(\tilde{\mathbf{x}})\right| V^{-1}(\tilde{\mathbf{x}}) \quad .
$$

We now insert relation (60) into expression (58) and arrive at expression

$$
L(\mathbf{x}, \tilde{\mathbf{x}})=\sqrt{\frac{\left|\varepsilon_{i j k} X_{1}^{i}(\mathbf{x}) X_{2}^{j}(\mathbf{x}) V^{k}(\mathbf{x})\right| V(\tilde{\mathbf{x}})}{v(\mathbf{x})\left|\mathbf{Y}_{1}(\tilde{\mathbf{x}}) \times \mathbf{Y}_{2}(\tilde{\mathbf{x}})\right| v(\tilde{\mathbf{x}})}}
$$

(Chapman, 2004, Eq. 5.4.19) for the relative geometrical spreading.

For the special case of a homogeneous Hamiltonian function, we may insert relation (26) into expression (61). In this case,

$$
L(\mathbf{x}, \tilde{\mathbf{x}})=\sqrt{\frac{\left|\mathbf{X}_{1}(\mathbf{x}) \times \mathbf{X}_{2}(\mathbf{x})\right|}{\left|\mathbf{Y}_{1}(\tilde{\mathbf{x}}) \times \mathbf{Y}_{2}(\tilde{\mathbf{x}})\right|} \frac{V(\tilde{\mathbf{x}})}{v(\tilde{\mathbf{x}})}} .
$$

This equation is not applicable to a general Hamiltonian function. Expression (62) with special initial conditions $\left|\mathbf{Y}_{1}(\tilde{\mathbf{x}}) \times \mathbf{Y}_{2}(\tilde{\mathbf{x}})\right|=V(\tilde{\mathbf{x}}) / v(\tilde{\mathbf{x}})$ was used by P̌senči $k$ and Teles (1996, Eqs A.1, A.4), Farra and Pšenč $\imath$ (2008, Eqs 25, 28) and Červený and Pšenčik (2015, Eq. 56).

5.4. Amplitude in terms of the propagator matrix of geodesic deviation in Cartesian coordinates

Paraxial vectors $X_{A}^{i}(\mathbf{x})$ and $Y_{m A}(\tilde{\mathbf{x}})$ corresponding to arbitrarily parametrized rays from a point source at $\tilde{\mathbf{x}}$ are related by equation

$$
X_{A}^{i}(\mathbf{x})=X_{2}^{i m}(\mathbf{x}, \tilde{\mathbf{x}}) Y_{m A}(\tilde{\mathbf{x}}),
$$

where $X_{2}^{i m}(\mathbf{x}, \tilde{\mathbf{x}})$ is the $3 \times 3$ upper right submatrix (19) of the $6 \times 6$ propagator matrix of geodesic deviation in Cartesian coordinates. Then

$$
\varepsilon_{i j k} X_{1}^{i}(\mathbf{x}) X_{2}^{j}(\mathbf{x}) V^{k}(\mathbf{x})=\varepsilon_{i j k} X_{2}^{i m}(\mathbf{x}, \tilde{\mathbf{x}}) X_{2}^{j n}(\mathbf{x}, \tilde{\mathbf{x}}) V^{k}(\mathbf{x}) Y_{m 1}(\tilde{\mathbf{x}}) Y_{n 2}(\tilde{\mathbf{x}}) \quad .
$$

We consider the skewness of Levi-Civita symbol $\varepsilon_{i j k}$, and rewrite relation (64) to read

$$
\begin{aligned}
& \varepsilon_{i j k} X_{1}^{i}(\mathbf{x}) X_{2}^{j}(\mathbf{x}) V^{k}(\mathbf{x}) \\
& \quad=\frac{1}{2} \varepsilon_{i j k} X_{2}^{i m}(\mathbf{x}, \tilde{\mathbf{x}}) X_{2}^{j n}(\mathbf{x}, \tilde{\mathbf{x}}) V^{k}(\mathbf{x})\left[Y_{m 1}(\tilde{\mathbf{x}}) Y_{n 2}(\tilde{\mathbf{x}})-Y_{n 1}(\tilde{\mathbf{x}}) Y_{m 2}(\tilde{\mathbf{x}})\right] .
\end{aligned}
$$


We now insert identity

$$
\varepsilon_{m n l} \varepsilon^{l r s}=\delta_{m r} \delta_{n s}-\delta_{m s} \delta_{n r}
$$

into relation (65), and arrive at

$$
\varepsilon_{i j k} X_{1}^{i}(\mathbf{x}) X_{2}^{j}(\mathbf{x}) V^{k}(\mathbf{x})=\frac{1}{2} \varepsilon_{i j k} X_{2}^{i m}(\mathbf{x}, \tilde{\mathbf{x}}) X_{2}^{j n}(\mathbf{x}, \tilde{\mathbf{x}}) V^{k}(\mathbf{x}) \varepsilon_{m n l} \varepsilon^{l r s} Y_{r 1}(\tilde{\mathbf{x}}) Y_{s 2}(\tilde{\mathbf{x}})
$$

We insert relation (59) into relation (67), and obtain relation

$$
\begin{aligned}
& \varepsilon_{i j k} X_{1}^{i}(\mathbf{x}) X_{2}^{j}(\mathbf{x}) V^{k}(\mathbf{x}) \\
& \quad= \pm \frac{1}{2} \varepsilon_{i j k} X_{2}^{i m}(\mathbf{x}, \tilde{\mathbf{x}}) X_{2}^{j n}(\mathbf{x}, \tilde{\mathbf{x}}) V^{k}(\mathbf{x}) \varepsilon_{m n l}\left|\mathbf{Y}_{1}(\tilde{\mathbf{x}}) \times \mathbf{Y}_{2}(\tilde{\mathbf{x}})\right| V^{l}(\tilde{\mathbf{x}})[V(\tilde{\mathbf{x}})]^{-1} .
\end{aligned}
$$

We define the matrix

$$
C_{k l}(\mathbf{x}, \tilde{\mathbf{x}})=\frac{1}{2} \varepsilon_{k i j} \varepsilon_{l m n} X_{2}^{i m}(\mathbf{x}, \tilde{\mathbf{x}}) X_{2}^{j n}(\mathbf{x}, \tilde{\mathbf{x}})
$$

of the cofactors of matrix $X_{2}^{i m}(\mathbf{x}, \tilde{\mathbf{x}})$, insert relation (68) with definition (69) into expression (61), and obtain relation

$$
L(\mathbf{x}, \tilde{\mathbf{x}})=\sqrt{\frac{\left|V^{k}(\mathbf{x}) C_{k l}(\mathbf{x}, \tilde{\mathbf{x}}) V^{l}(\tilde{\mathbf{x}})\right|}{v(\mathbf{x}) v(\tilde{\mathbf{x}})}}
$$

(Kendall et al., 1992, Eq. 17b; Chapman, 2004, Eq. 5.4.23) for the relative geometrical spreading.

5.5. Amplitude in terms of the second-order derivatives of the characteristic function in ray-centred coordinates

Klimě̌ (2013a, Eq. 28) derived relation

$$
\left(\frac{\partial^{2} \tau}{\partial \tilde{x}^{i} \partial x^{j}}+\frac{1}{\Gamma} \frac{\partial \gamma}{\partial \tilde{x}^{i}} \frac{\partial \gamma}{\partial x^{j}}\right)(\mathbf{x}, \tilde{\mathbf{x}}) X_{2}^{j k}(\mathbf{x}, \tilde{\mathbf{x}})=-\delta_{i}^{k}
$$

between the mixed second-order spatial derivatives of characteristic function $\tau\left(\mathbf{x}, \mathbf{x}^{\prime}\right)$ and the $3 \times 3$ upper right submatrix of the $6 \times 6$ propagator matrix of geodesic deviation in general coordinates including Cartesian coordinates. The meaning of functions $\gamma(\mathbf{x}, \tilde{\mathbf{x}})$ and $\Gamma(\mathbf{x}, \tilde{\mathbf{x}})$ is not significant here. Interested readers may refer to Klimers $(2013 a, b)$.

If we define matrix $\left[X_{2}^{-1}\right]_{j i}(\mathbf{x}, \tilde{\mathbf{x}})$ inverse to matrix $X_{2}^{i j}(\mathbf{x}, \tilde{\mathbf{x}})$, we may express relation (71) as

$$
\left(\frac{\partial^{2} \tau}{\partial \tilde{x}^{i} \partial x^{j}}+\frac{1}{\Gamma} \frac{\partial \gamma}{\partial \tilde{x}^{i}} \frac{\partial \gamma}{\partial x^{j}}\right)(\mathbf{x}, \tilde{\mathbf{x}})=-\left[X_{2}^{-1}\right]_{i j}(\mathbf{x}, \tilde{\mathbf{x}})
$$

We now transform relation (72) into ray-centred coordinates.

We transform the submatrix $X_{2}^{i j}(\mathbf{x}, \tilde{\mathbf{x}})$ of the propagator matrix of geodesic deviation from Cartesian to ray-centred coordinates,

$$
Q_{2}^{a b}(\mathbf{x}, \tilde{\mathbf{x}})=\hat{h}_{i}^{a}(\mathbf{x}) X_{2}^{i j}(\mathbf{x}, \tilde{\mathbf{x}}) \hat{h}_{j}^{b}(\tilde{\mathbf{x}})
$$




\section{Klimes}

The transformation of the inverse matrix then reads

$$
\left[Q_{2}^{-1}\right]_{b a}(\mathbf{x}, \tilde{\mathbf{x}})=h_{b}^{j}(\tilde{\mathbf{x}})\left[X_{2}^{-1}\right]_{j i}(\mathbf{x}, \tilde{\mathbf{x}}) h_{a}^{i}(\mathbf{x}) \quad .
$$

We transform the mixed second-order derivatives of the characteristic function from Cartesian to ray-centred coordinates,

$$
\frac{\partial^{2} \tau}{\partial q^{a} \partial \tilde{q}^{b}}(\mathbf{x}, \tilde{\mathbf{x}})=\frac{\partial x^{k}}{\partial q^{a}}(\mathbf{x}) \frac{\partial^{2} \tau}{\partial x^{k} \partial \tilde{x}^{l}}(\mathbf{x}, \tilde{\mathbf{x}}) \frac{\partial \tilde{x}^{l}}{\partial \tilde{q}^{b}}(\tilde{\mathbf{x}})
$$

which can be expressed in terms of the contravariant basis vectors (8) of the raycentred coordinate system as

$$
\frac{\partial^{2} \tau}{\partial q^{a} \partial \tilde{q}^{b}}(\mathbf{x}, \tilde{\mathbf{x}})=h_{a}^{k}(\mathbf{x}) \frac{\partial^{2} \tau}{\partial x^{k} \partial \tilde{x}^{l}}(\mathbf{x}, \tilde{\mathbf{x}}) h_{b}^{l}(\tilde{\mathbf{x}})
$$

Since

$$
\frac{\partial H}{\partial p_{k}}(\mathbf{x}) \frac{\partial^{2} \tau}{\partial x^{k} \partial \tilde{x}^{l}}(\mathbf{x}, \tilde{\mathbf{x}})=0
$$

(Hamilton, 1837, Eqs U, I), and

$$
\frac{\partial^{2} \tau}{\partial x^{k} \partial \tilde{x}^{l}}(\mathbf{x}, \tilde{\mathbf{x}}) \frac{\partial H}{\partial p_{l}}(\tilde{\mathbf{x}})=0
$$

(Hamilton, 1837, Eqs Y, I), we have identities

$$
\frac{\partial^{2} \tau}{\partial q^{A} \partial \tilde{q}^{3}}(\mathbf{x}, \tilde{\mathbf{x}})=0 \quad, \quad \frac{\partial^{2} \tau}{\partial q^{3} \partial \tilde{q}^{B}}(\mathbf{x}, \tilde{\mathbf{x}})=0
$$

Relation (72) in ray-centred coordinates then reads

$$
\left[Q_{2}^{-1}\right]_{b a}(\mathbf{x}, \tilde{\mathbf{x}})=-\left(\begin{array}{cc}
\frac{\partial^{2} \tau}{\partial \tilde{q}^{B} \partial q^{A}}+\frac{\partial \gamma}{\partial \tilde{q}^{B}} \frac{1}{\Gamma} \frac{\partial \gamma}{\partial q^{A}} & \frac{\partial \gamma}{\partial \tilde{q}^{B}} \frac{1}{\Gamma} \frac{\partial \gamma}{\partial q^{3}} \\
\frac{\partial \gamma}{\partial \tilde{q}^{3}} \frac{1}{\Gamma} \frac{\partial \gamma}{\partial q^{A}} & \frac{\partial \gamma}{\partial \tilde{q}^{3}} \frac{1}{\Gamma} \frac{\partial \gamma}{\partial q^{3}}
\end{array}\right)(\mathbf{x}, \tilde{\mathbf{x}})
$$

We define $2 \times 2$ matrix

$$
Q_{2}^{B A}(\mathbf{x}, \tilde{\mathbf{x}})=-\left(\frac{\partial^{2} \tau}{\partial \tilde{q}^{A} \partial q^{B}}\right)^{-1}(\mathbf{x}, \tilde{\mathbf{x}})
$$

invert the $3 \times 3$ matrices in relation (80), and arrive at

$$
Q_{2}^{a b}(\mathbf{x}, \tilde{\mathbf{x}})=\left(\begin{array}{cc}
Q_{2}^{A B} & -Q_{2}^{A D} \frac{\partial \gamma}{\partial \tilde{q}^{D}}\left(\frac{\partial \gamma}{\partial \tilde{q}^{3}}\right)^{-1} \\
-\frac{\partial \gamma}{\partial q^{C}} Q_{2}^{C B}\left(\frac{\partial \gamma}{\partial q^{3}}\right)^{-1} & \left(\Gamma+\frac{\partial \gamma}{\partial q^{C}} Q_{2}^{C D} \frac{\partial \gamma}{\partial \tilde{q}^{D}}\right)\left(\frac{\partial \gamma}{\partial q^{3}} \frac{\partial \gamma}{\partial \tilde{q}^{3}}\right)^{-1}
\end{array}\right)(\mathbf{x}, \tilde{\mathbf{x}})
$$

We see that the $2 \times 2$ matrix $Q_{2}^{B A}(\mathbf{x}, \tilde{\mathbf{x}})$ is a submatrix of the $3 \times 3$ matrix (73). Definition (81) may then be understood as the relation between the $2 \times 2$ submatrix $Q_{2}^{B A}(\mathbf{x}, \tilde{\mathbf{x}})$ of the $3 \times 3$ matrix (73) and the mixed second-order spatial derivatives of characteristic function $\tau\left(\mathbf{x}, \mathbf{x}^{\prime}\right)$ in ray-centred coordinates. This relation represents a generalization of the relation for the mixed second-order spatial derivatives of characteristic function $\tau\left(\mathbf{x}, \mathbf{x}^{\prime}\right)$ by $\breve{C}$ ervený et al. (1984, Eq. 22) to anisotropic media. 
Relation (81) yields relation

$$
\operatorname{det}\left(\frac{\partial^{2} \tau}{\partial q^{A} \partial \tilde{q}^{B}}(\mathbf{x}, \tilde{\mathbf{x}})\right)=-1 / \operatorname{det}\left[\mathbf{Q}_{2}(\mathbf{x}, \tilde{\mathbf{x}})\right] .
$$

We insert relation (83) into expression (39) and obtain expression

$$
L(\mathbf{x}, \tilde{\mathbf{x}})=\sqrt{\left|\mathbf{h}_{1}(\mathbf{x}) \times \mathbf{h}_{2}(\mathbf{x})\right|\left|\operatorname{det}\left(\frac{\partial^{2} \tau}{\partial q^{A} \partial \tilde{q}^{B}}(\mathbf{x}, \tilde{\mathbf{x}})\right)\right|^{-1}\left|\mathbf{h}_{1}(\tilde{\mathbf{x}}) \times \mathbf{h}_{2}(\tilde{\mathbf{x}})\right|}
$$

for the relative geometrical spreading in terms of the mixed second-order spatial derivatives of the characteristic function in ray-centred coordinates. The special case of expression (84), corresponding to orthonormal contravariant basis vectors $\mathbf{h}_{1}$ and $\mathbf{h}_{2}$, was presented by Schleicher et al. (2001, Eqs 5-6).

Expression (84) for the relative geometrical spreading may also be modified by inserting identity (47), both at point $\tilde{\mathbf{x}}$ or point $\mathbf{x}$, e.g.,

$$
L(\mathbf{x}, \tilde{\mathbf{x}})=\sqrt{\frac{V(\mathbf{x})}{v(\mathbf{x})} \frac{V(\tilde{\mathbf{x}})}{v(\tilde{\mathbf{x}})}} / \sqrt{\left|\hat{\mathbf{h}}^{1}(\mathbf{x}) \times \hat{\mathbf{h}}^{2}(\mathbf{x})\right|\left|\operatorname{det}\left(\frac{\partial^{2} \tau}{\partial q^{A} \partial \tilde{q}^{B}}(\mathbf{x}, \tilde{\mathbf{x}})\right)\right|\left|\hat{\mathbf{h}}^{1}(\tilde{\mathbf{x}}) \times \hat{\mathbf{h}}^{2}(\tilde{\mathbf{x}})\right|} .
$$

5.6. Amplitude in terms of the second-order derivatives of the characteristic function in Cartesian coordinates

The transformation of the mixed second-order derivatives of the characteristic function from ray-centred to Cartesian coordinates reads

$$
\frac{\partial^{2} \tau}{\partial x^{k} \partial \tilde{x}^{l}}(\mathbf{x}, \tilde{\mathbf{x}})=\frac{\partial q^{a}}{\partial x^{k}}(\mathbf{x}) \frac{\partial^{2} \tau}{\partial q^{a} \partial \tilde{q}^{b}}(\mathbf{x}, \tilde{\mathbf{x}}) \frac{\partial \tilde{q}^{b}}{\partial \tilde{x}^{l}}(\tilde{\mathbf{x}})
$$

Considering identities (79), we express relation (86) in terms of the covariant basis vectors (10) of the ray-centred coordinate system as

$$
\frac{\partial^{2} \tau}{\partial x^{j} \partial \tilde{x}^{m}}(\mathbf{x}, \tilde{\mathbf{x}})=\hat{h}_{j}^{A}(\mathbf{x}) \frac{\partial^{2} \tau}{\partial q^{A} \partial \tilde{q}^{B}}(\mathbf{x}, \tilde{\mathbf{x}}) \hat{h}_{m}^{B}(\tilde{\mathbf{x}})
$$

We define the matrix

$$
W^{i l}(\mathbf{x}, \tilde{\mathbf{x}})=\frac{1}{2} \varepsilon^{i j k} \varepsilon^{l m n} \frac{\partial^{2} \tau}{\partial x^{j} \partial \tilde{x}^{m}}(\mathbf{x}, \tilde{\mathbf{x}}) \frac{\partial^{2} \tau}{\partial x^{k} \partial \tilde{x}^{n}}(\mathbf{x}, \tilde{\mathbf{x}})
$$

of the cofactors of matrix (86). We insert relation (87) into definition (88) and arrive at relation

$$
\begin{aligned}
W^{i l}(\mathbf{x}, \tilde{\mathbf{x}}) & =\varepsilon^{i j k} \hat{h}_{j}^{1}(\mathbf{x}) \hat{h}_{k}^{2}(\mathbf{x}) \varepsilon^{l m n} \hat{h}_{m}^{1}(\tilde{\mathbf{x}}) \hat{h}_{n}^{2}(\tilde{\mathbf{x}}) \\
& \times\left[\frac{\partial^{2} \tau}{\partial q^{1} \partial \tilde{q}^{1}}(\mathbf{x}, \tilde{\mathbf{x}}) \frac{\partial^{2} \tau}{\partial q^{2} \partial \tilde{q}^{2}}(\mathbf{x}, \tilde{\mathbf{x}})-\frac{\partial^{2} \tau}{\partial q^{1} \partial \tilde{q}^{2}}(\mathbf{x}, \tilde{\mathbf{x}}) \frac{\partial^{2} \tau}{\partial q^{2} \partial \tilde{q}^{1}}(\mathbf{x}, \tilde{\mathbf{x}})\right] .
\end{aligned}
$$

We insert relation (42) into relation (89) and obtain relation

$$
W^{i l}(\mathbf{x}, \tilde{\mathbf{x}})= \pm\left|\hat{\mathbf{h}}^{1}(\mathbf{x}) \times \hat{\mathbf{h}}^{2}(\mathbf{x})\right| \frac{V^{i}(\mathbf{x})}{V(\mathbf{x})}\left|\hat{\mathbf{h}}^{1}(\tilde{\mathbf{x}}) \times \hat{\mathbf{h}}^{2}(\tilde{\mathbf{x}})\right| \frac{V^{l}(\tilde{\mathbf{x}})}{V(\tilde{\mathbf{x}})} \operatorname{det}\left(\frac{\partial^{2} \tau}{\partial q^{A} \partial \tilde{q}^{B}}(\mathbf{x}, \tilde{\mathbf{x}})\right) .
$$




\section{Kliměs}

We multiply relation (90) by $p_{i}(\mathbf{x})$ and $p_{l}(\tilde{\mathbf{x}})$, insert the product into expression (85), and obtain expression

$$
L(\mathbf{x}, \tilde{\mathbf{x}})=1 / \sqrt{\left|p_{i}(\mathbf{x}) W^{i l}(\mathbf{x}, \tilde{\mathbf{x}}) p_{l}(\tilde{\mathbf{x}})\right| v(\mathbf{x}) v(\tilde{\mathbf{x}})} .
$$

for the relative geometrical spreading in terms of the mixed second-order derivatives of the characteristic function in Cartesian coordinates.

We insert expression (91) for the relative geometrical spreading into expression (38) and obtain new expression

$$
A^{\mathrm{G}}(\mathbf{x}, \tilde{\mathbf{x}})=\frac{1}{4 \pi} \sqrt{\frac{\left|p_{i}(\mathbf{x}) W^{i l}(\mathbf{x}, \tilde{\mathbf{x}}) p_{l}(\tilde{\mathbf{x}})\right|}{\varrho(\mathbf{x}) \varrho(\tilde{\mathbf{x}})}} \exp [\mathrm{i} \varphi(\mathbf{x}, \tilde{\mathbf{x}})]
$$

for the amplitude of the Green tensor.

\section{CONCLUSIONS}

The zero-order ray-theory amplitude satisfies the transport equation. There are two important kinds of the ray-theory amplitude: the amplitude corresponding to a wavefield with general initial conditions including finite sources, and the amplitude of the Green tensor. Thre already known equivalent expressions (24), (25) and (37) for the amplitude corresponding to the general initial conditions are presented in Section 4. Relation (38) for the amplitude of the Green tensor is presented in Section 5 together with twelve equivalent expressions for the relative geometrical spreading. Expressions (39), (58), (61) and (70) for the relative geometrical spreading have already been published. Expressions (48), (49), (51), (52), (53), (84), (85) and (91) for the relative geometrical spreading may be novel, although some special cases of some of these expressions have been published. The step-by-step derivation of various equivalent expressions starts with expressions (24) and (25), or expressions (38) and (39), respectively. The derivation thus elucidates the mutual relations between the equivalent expressions.

The Hamiltonian equations of geodesic deviation (paraxial ray equations, dynamic ray tracing equations) are sometimes expressed and solved in general Cartesian coordinates and sometimes in ray-centred coordinates connected with a particular ray. The expressions for the zero-order ray-theory amplitude in terms of the results of the Hamiltonian equations of geodesic deviation in general Cartesian coordinates are presented in Sections 4.1, 5.3, 5.4 and 5.6. The expressions for the zero-order ray-theory amplitude in terms of the results of the Hamiltonian equations of geodesic deviation in ray-centred coordinates are presented in Sections 4.2, 5.1, 5.2 and 5.5 .

The zero-order ray-theory amplitude of the Green tensor can be expressed in terms of the propagator matrix of the Hamiltonian equations of geodesic deviation (Sections 5.1 and 5.4), in terms of the paraxial vectors corresponding to arbitrarily parametrized rays from a point source (Sections 5.2 and 5.3), or in terms of the second-order derivatives of the characteristic function (Sections 5.5 and 5.6). 
Acknowledgements: The author is indebted to Vlastislav Červený for many invaluable discussions on the topic of this paper. The research has been supported by the Grant Agency of the Czech Republic under contract 16-05237S, and by the members of the consortium "Seismic Waves in Complex 3-D Structures" (see "http://sw3d.cz").

\section{References}

Babich V.M., 1961. Ray method of calculating the intensity of wavefronts in the case of a heterogeneous, anisotropic, elastic medium. In: Petrashen G.I. (Ed.), Problems of the Dynamic Theory of Propagation of Seismic Waves, Vol. 5. Leningrad Univ. Press, Leningrad, 36-46 (in Russian, English translation: Geophys. J. Int., 118(1994), 379-383).

Bakker P.M., 1998. Phase shift at caustics along rays in anisotropic media. Geophys. J. Int., 134, 515-518.

Červený V., 1972. Seismic rays and ray intensities in inhomogeneous anisotropic media. Geophys. J. R. Astr. Soc., 29, 1-13.

Červený V., 2001. Seismic Ray Theory. Cambridge Univ. Press, Cambridge, U.K.

Červený V., Klimeš L. and Pšenčík I., 1984. Paraxial ray approximations in the computation of seismic wavefields in inhomogeneous media. Geophys. J. R. Astr. Soc., 79, 89-104.

Červený V., Klimeš L. and Pšenčík I., 1988. Complete seismic-ray tracing in threedimensional structures. In: Doornbos D.J. (Ed.), Seismological Algorithms. Academic Press, New York, 89-168.

Červený V. and Pšenčík I., 2014. Summation integrals for a Green function in a 3-D inhomogeneous anisotropic medium. Seismic Waves in Complex 3-D Structures, 24, 131-158 (http://sw3d.cz).

Červený V. and Pšenčík I., 2015. Integral superposition of paraxial Gaussian beams in inhomogeneous anisotropic layered structures in Cartesian coordinates. Seismic Waves in Complex 3-D Structures, 25, 109-155 (http://sw3d.cz).

Chapman C.H., 2004. Fundamentals of Seismic Wave Propagation. Cambridge Univ. Press, Cambridge, U.K.

Farra V. and Pšenčík I., 2008. First-order ray computations of coupled S waves in inhomogeneous weakly anisotropic media. Geophys. J. Int., 173, 979-989.

Gajewski D. and Pšenčík I., 1990. Vertical seismic profile synthetics by dynamic ray tracing in laterally varying layered anisotropic structures. J. Geophys. Res., 95B, 11301-11315.

Garmany J., 2001. Phase shifts at caustics in anisotropic media. In: Ikelle L. and Gangi A. (Eds), Anisotropy 2000: Fractures, Converted Waves and Case Studies. Soc. Explor. Geophysicists, Tulsa, 419-425.

Hamilton W.R., 1837. Third supplement to an essay on the theory of systems of rays. Trans. Roy. Irish Acad., 17, 1-144.

Kendall J-M., Guest W.S. and Thomson C.J., 1992. Ray-theory Green's function reciprocity and ray-centred coordinates in anisotropic media. Geophys. J. Int., 108, 364371. 


\section{Kliměs}

Kendall J-M. and Thomson C.J., 1989. A comment on the form of geometrical spreading equations, with some examples of seismic ray tracing in inhomogeneous, anisotropic media. Geophys. J. Int., 99, 401-413.

Klimeš L., 1994. Transformations for dynamic ray tracing in anisotropic media. Wave Motion, 20, 261-272.

Klimeš L., 2006a. Spatial derivatives and perturbation derivatives of amplitude in isotropic and anisotropic media. Stud. Geophys. Geod., 50, 417-430.

Klimeš L., 2006b. Ray-centred coordinate systems in anisotropic media. Stud. Geophys. Geod., 50, 431-447.

Klimeš L., 2010. Phase shift of the Green tensor due to caustics in anisotropic media. Stud. Geophys. Geod., 54, 268-289.

Klimeš L., 2012. Zero-order ray-theory Green tensor in a heterogeneous anisotropic elastic medium. Stud. Geophys. Geod., 56, 373-382.

Klimeš L., 2013a. Relation between the propagator matrix of geodesic deviation and the second-order derivatives of the characteristic function for a general Hamiltonian function. Seismic Waves in Complex 3-D Structures, 23, 121-134 (http://sw3d.cz).

Klimeš L., 2013b. Calculation of the spatial gradient of the independent parameter along geodesics for a general Hamiltonian function. Seismic Waves in Complex 3-D Structures, 23, 135-143 (http://sw3d.cz).

Klimeš L., 2014. Phase shift of a general wavefield due to caustics in anisotropic media. Seismic Waves in Complex 3-D Structures, 24, 95-109 (http://sw3d.cz).

Kravtsov Yu.A. and Orlov Yu.I., 1993. Caustics, Catastrophes and Wave Fields. Springer, Berlin-Heidelberg, Germany.

Kravtsov Yu.A. and Orlov Yu.I., 1999. Caustics, Catastrophes and Wave Fields. Springer, Berlin-Heidelberg, Germany.

Lewis R.M., 1965. Asymptotic theory of wave-propagation. Arch. Ration. Mech. Anal., 20, 191-250.

Moser T.J. and Červený V., 2007. Paraxial ray methods for anisotropic inhomogeneous media. Geophys. Prospect., 55, 21-37.

Orlov Yu.I., 1981. Caustics with anomalous phase shifts. Radiophys. Quantum Electron., 24, 154-159.

Pšenčík I. and Teles T.N., 1996. Point-source radiation in inhomogeneous anisotropic structures. Pure Appl. Geophys., 148, 591-623.

Schleicher J., Tygel M., Ursin B. and Bleistein N., 2001. The Kirchhoff-Helmholtz integral for anisotropic elastic media. Wave Motion, 34, 353-364. 